International

Medical Society

http://imedicalsociety.org
International Archives of Medicine

SECTION: SPORTS MEDICINE ISSN: 1755-7682
2019

Vol. 12 No. 19

doi: $10.3823 / 2613$

\title{
Sports Rehabilitation and Interprofessional Collaboration
}

Nicholas B. Washmuth ${ }^{1,2}$, James M. Sepich ${ }^{3}$, Abby D. McAfee ${ }^{4}$

1 Department of Physical Therapy, Samford University, 800 Lakeshore Dr, Homewood, AL 35229.

\section{Abstract}

Background: The demand for interprofessional collaboration continues to grow due to changing priorities of the health care system. The benefits of interprofessional collaboration are well documented; however, the methods of collaboration are often unclear and often difficult to put into practice. While there is a growing number of studies on the impact of interprofessional collaboration in settings such as inpatient, intensive care units, and acute care, there are limited documented cases regarding the interprofessional management of a physical therapy patient in the outpatient orthopedic private practice setting.

Purpose: The purpose of this paper is to describe the reflections of a physical therapist and an athletic trainer in their experience with interprofessional collaboration, describe the barriers they have experienced that make interprofessional collaboration challenging, and to offer solutions to these barriers. The barriers discussed in the paper include limited knowledge of and respect for other professionals' skill set, high-productivity work environments, medical hierarchy, overlapping bodies of knowledge, discrepancy between professional reasoning, territorial behavior, and ineffective communication.

Discussion: It appears that contextual factors, such as community in which the professionals practice, the healthcare setting, and the practice environment, have a far less important impact to successful collaboration than the professionals' attitudes and investment in the collaborative efforts.

Conclusion: Collaboration between a PT and an AT can lead to power struggles and suboptimal patient care if these barriers are not overcome and collaboration may be necessary to provide the highest quality of patient care.

2 PhysioFit, Homewood, AL, 35209.

3 ATI Physical Therapy, 1000 Eleven South, Ste 1B, Columbia, IL 62236.

4 Alton Physical Therapy, 1719 Clawson, Alton, IL 62002.

\section{Contact information:}

\section{Nicholas B. Washmuth.}

Address: Samford University. Department of Physical Therapy. 800 Lakeshore Dr. Homewood, AL 35229.

Tel: 314-229-8043.

ほnwashmut@samford.edu
Keywords
Interprofessional,
Collaboration, Barriers,
Physical Therapy, Athletic
Training. 


\section{Introduction}

\section{Background}

The changing organizational structures, reimbursement models, and priorities of the health care system are creating demands for interprofessional collaboration. There is a paradigm shift occurring in how health care services are being reimbursed. The traditional fee for service method, where a provider is reimbursed based on the services they provide, may no longer apply. A value based reimbursement system, consisting of being reimbursed based on the quality of the patient's outcomes, is the future of health care reimbursement [1]. These characteristics necessitate a team approach to health care delivery that encourages effective interprofessional communication and collaboration. Interprofessional collaborative practice can be defined as pursuing or engaging in a two-way exchange of information between professionals that is conducive to developing collaborative working relationships [2]. The purpose of interprofessional collaboration is to bring a broader scope of expertise to the patient in an effort to improve the quality of care [3] and patient outcomes.

Interprofessional collaboration is increasingly recognized as a means of improving patient outcomes and the cost effectiveness of care in a variety of settings from primary medical care to acute care to outpatient rehabilitation [4]. The improved quality of care, patient outcomes, and cost effectiveness with interprofessional collaboration is attributed to adherence to recommended practices, improved use of healthcare resources, and improved team communication and coordination [5]. The concept of interprofessional collaboration has been around for decades [6] but has become more necessary in health care because of the increasing specialization of services and professionalization of multiple health care disciplines [7]. The needs of most patients are beyond the expertise of any single profession but seeing multiple specialists can lead to a fragmentation of services provided for different patients [7].

Improved collaborative practice can play a significant role in mitigating many of the challenges faced by the health care system. Interprofessional collaboration, when conducted properly, can optimize the skills of each professional, share case management, and provide better services to patients [8]. Well-coordinated collaboration across health care professions has the potential to allow comprehensive and cost-effective care [9], which will help to meet the contemporary health care challenges. Workforce-diversity consultants have consistently stated that collaboration through diverse workgroups promotes new ideas, goals, and practices that may benefit the greater good of an organization, profession, or group of people [10], such as the patient in the health care setting. There is a growing body of evidence that suggests error rates increase and patients experience poor outcomes when ineffective communication exists between health professionals [2]. Therefore, improving interprofessional communication and collaboration may result in a strengthened health care system with improved outcomes $[2,3]$.

The benefits of interprofessional collaboration is well documented; however, the methods of collaboration are often unclear and often difficult to put into practice. While there is a growing number of studies on the impact of interprofessional collaboration on patient outcomes in fields and settings such as stroke [2], secondary care [2], inpatient care [2], geriatrics [2, 3], intensive care units [3], and acute care [2], there has been no documented cases, to the authors' knowledge, regarding the interprofessional management of a physical therapy patient in the outpatient orthopedic setting. The focus of past research has been on collaboration among a narrow range of health professionals, namely, physicians and nurses [3]. The collaboration contributions of a broader array of health professionals to outcomes of care need to be documented. 


\section{Specific aims}

The purpose of this paper is to describe the reflections of a physical therapist and an athletic trainer in their experience with interprofessional collaboration and to offer guidance to rehabilitation professionals with a similar scope of practice. The Samford University Institutional Review Board provided approval for this project, approval number EXPD-HP-15-SUM-01.

\section{Description of the traditional role of the physical therapist and athletic trainer}

The general scope of professional practice for health professionals like physical therapist and athletic trainers includes the evaluation and treatment of the patient as well as significant communication with the patient's family and other health care providers. Physical therapists (PTs) routinely collaborate with physicians, interact with other allied health professionals, supervise assistant personnel, and coordinate services across the health care continuum. The scopes of practice of PTs and athletic trainers (ATs) overlap in many areas, which allow these professionals to work with similar patient populations. According to the National Athletic Trainers' Association, athletic trainers are health care professionals who collaborate with physicians to provide preventative services, emergency care, clinical diagnosis, therapeutic intervention and rehabilitation of injuries and medical conditions. Similarly, according to the American Physical Therapy Association, physical therapists are health care professionals who help individuals maintain, restore, and improve movement, activity, and functioning, thereby enabling optimal performance and enhancing health, well-being, and quality of life. In order for a medical specialty, such as physical therapy and athletic training, to improve its professional skills and competencies, the medical specialty must define its field.

Interprofessional collaboration between a PT and an AT requires a commitment from practitioners to work together across traditional boundaries for the benefit of the patient. Improved patient-client care and outcomes can be accomplished by playing up to each discipline's core competencies and each clinicians' specific skill set. Despite the commonalities of ATs and PTs, the guidance available to the clinician for collaboration is limited [11]. As physical therapists and athletic trainers may vie to treat the same patient, especially in an athletic or outpatient orthopedic patient setting, the competition between these professions has increased.

\section{Description of practice setting}

Just as the scope of practice for PTs and ATs overlap in many areas, the practice settings in which these professionals practice are often very similar. PTs working in a private practice outpatient orthopedic setting may be working in close proximity to their AT colleagues. ATs who work in a sports training room may be working in close proximity to their PT colleagues. The authors have experience working in private practice physical therapy clinical settings in which the primary patient population is general orthopedic musculoskeletal, where the majority of the patients are referred to the clinic by physicians and ATs, and to a lesser extent by chiropractors, dentists, and direct access.

\section{Discussion}

\section{Reflections}

The discussion below will highlight the barriers (Figure 1) experienced from an AT and a PT when collaborating and offer insight to other health care professionals on how to overcome the barriers to operate successfully in a collaborative environment. The strategies employed by these clinicians to overcome the constraints to collaboration may hold valuable lessons for other outpatient orthopedic rehabilitation clinics as well as other settings in which care is delivered by a diverse and changing mix of professionals in the face of heavy patient demands. 
Figure 1: Barriers to interprofessional collaboration

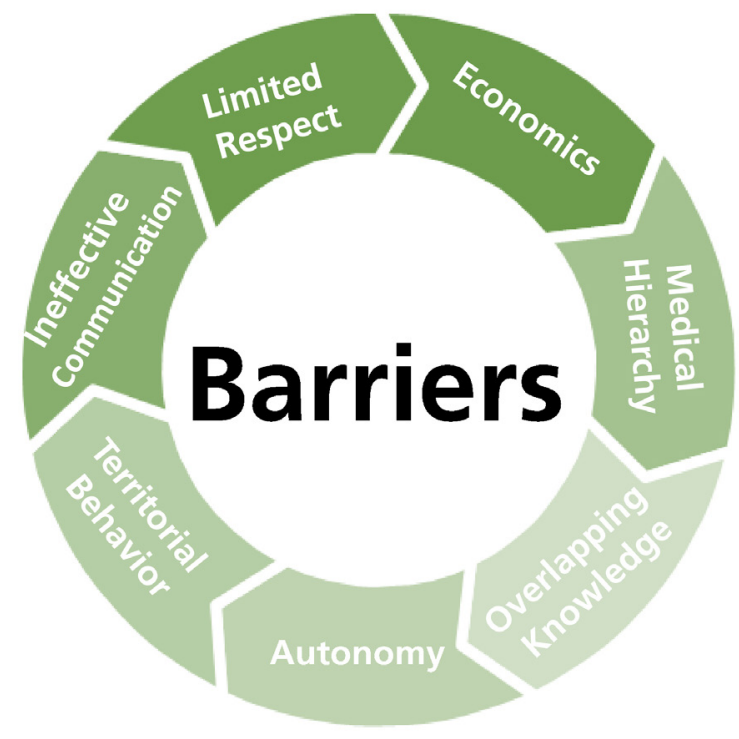

\section{Barrier 1}

\section{Limited knowledge of and respect for other professionals' skill set}

Health care professionals often have a limited understanding of their colleagues' skills and competencies related to patient care [2]. It has been previously reported that PTs and ATs often do not understand each other's roles and; therefore, do not acknowledge or respect what the other professional has to offer a patient [12]. This limited understanding of other professionals' skills and competencies leads to role ambiguity and being unaware of the information or need for information that the other provider has regarding a specific patient [2]. This is especially true for professionals with scopes of practice that can be very similar, such as a PT and $A T$, and a nurse and physician.

\section{Solution}

Respect and trust are seen as important elements of effective collaboration among different professionals working with the same patient [11]. The PT and AT sought out opportunities to talk routinely with other PTs, ATs, and physicians to not only identify and solve patient care problems but to learn about each profession's body of knowledge and professional reasoning. By offering ideas and opinions using each professional's rationale allowed the opportunity to learn about each provider's skill set. Another method the PT and AT used to foster respect was to acknowledge working with and voicing gratitude about each other when talking with other providers. This allows other professionals, including other PTs and ATs who lack experience working collaboratively, to learn about and understand what PTs and ATs have to offer.

It was our experience that interprofessional coIlaboration was a catalyst for professional development [6]. The PT and AT were able to recognize gaps in their practice when collaborating with each other and because of the mutual respect, were willing to learn and adapt their practice patterns. The PT and AT would frequently reach out to each other informally and ask for insight and advice on difficult cases, fostering mutual respect and trust.

\section{Barrier 2 \\ High-productivity work environments}

The productivity requirements of an outpatient physical therapy practice necessitate a fast-paced work environment. The economic realities of an evolving health care system warrant productivity standards for PTs. The fast-paced environment that is required to meet productivity standards is a barrier to developing and sustaining interprofessional relationships. The time it takes to initiate, develop, and maintain interprofessional relationships takes time away from revenue generating time with direct patient care. In fact, lack of time is reported to be a major factor for not participating in interprofessional collaborative meetings [13]. Healthcare is a business that must be profitable and sustainable which can create an environment that may indirectly discourage collaborative practices if it means losing a revenue stream due to decreasing productivity. 


\section{Solution}

Collaborative care may decrease revenue acutely. This decrease in revenue occurs when a clinician, in a setting that requires high productivity to sustain a business model, refers a patient to another provider outside of their organization, thereby decreasing productivity and revenue. The experience of the PT and AT supports that collaboration increases revenue long-term. It is with improved patient outcomes that foster trust and respect of other practitioners, which leads to more frequent referrals between providers allowing for more patient contacts and greater patient volume. It is also observed that patients respect the collaboration and being managed by the most appropriate provider.

Some data suggests that cost containment may be realized from interprofessional practice [5]; however, this data is collected from many different clinical settings and health conditions with mixed results. Costs are an inevitably important outcome to examine in the current climate of health care delivery. To our knowledge, there are no reports regarding the economics of collaborative practice in an outpatient rehabilitation private practice setting.

\section{Barrier 3}

\section{Medical hierarchy}

In traditional medical hierarchy, the physician can be accustomed to having their orders carried out with little or no discussion [2]. Subordinates to the physician may adopt various strategies to resist the hierarchical power structure, such as declining to participate in interprofessional meetings [2]. This resistance to traditional hierarchy disrupts the sharing of professional expertise, which is the foundation for interprofessional collaboration [3]. Although this traditional medical hierarchy can reduce collaborative work, it must be clear which professional encourages assignment of roles and responsibilities, as well as which professional has the authority to make final decisions related to each patient. The team leader with the authority to make final decisions must consistently communicate with all of the collaborative members of their health care team so that each member is clear on their individual roles and duties, as well as encouraging input from others and acknowledging contributions [11, 14]. If the medical hierarchy fails to do this, there is a decreased participation in the decision making and a disconnect in team ownership regarding patient care. The Athletic Training and Physical Therapy professions have expressed the importance of clarity about the decision-making, including a hierarchy of decisionmaking in certain situations [11]. It is routine that physicians make final decisions for complex cases, including return to play for athletes, usually with the input of both ATs and PTs [11].

\section{Solution}

Interprofessional collaboration between a PT and AT can occur at three different levels:

1) common, where treatments can be provided by either profession,

2) complimentary, where the uniqueness that distinguishes one profession from another can be utilized,

3) collaborative, where sharing occurs across both professions [4].

A patient-centered framework can be used to determine a leader who encourages roles and responsibilities of the other team members and who has the authority to make final decisions regarding the patient. At the beginning of each collaborative management of a patient, the PT and AT would assure there was a clear understanding of the framework being used for that specific episode of care, which allowed the collaboration to match roles and responsibilities of the clinician to the goals of the case, including accountable and proactive leadership. The PT and AT would often consider the original referral source of the patient and the goals of the patient in determining who will take the leadership role in a specific case. The leader should have the clinical judgment to determine when it is appropriate to 
discontinue care and have the clinical skill set required to ensure the patient reaches their goals. No matter who has the leadership role, the PT and AT realize that they could learn and grow through collaborative opportunities. The PT and AT remained humble throughout each collaborative case.

It has been hypothesized that the practice of interprofessional collaboration can take professionals out of their proverbial comfort zones [10]. In Physical Therapy and Athletic Training, specialty areas and professional distinctions, such as OCS (board-certified specialist in orthopedics) and SFMA (certification in selective functional movement assessment), often have created silos and egos, leading to arbitrary forms of distance and barriers, and at times, mistrust and lack of collegiately, not only within the physical therapy and athletic training professions, but interprofessionally. The leader of each collaborative effort must help prevent such silos from developing.

\section{Barrier 4}

\section{Overlapping bodies of knowledge}

Athletic Training and Physical Therapy professionals share a common understanding of the foundational sciences and a commitment to evidence based practice, but these two professionals can treat vastly different patient problems, treat patients at different stages of their rehabilitation, and have different treatment philosophies [2]. The collaborations between nurse practitioners and physicians share this same barrier of overlapping strengths that both disciplines may have, as they share knowledge, skills, beliefs and values regarding care delivery [15]. No matter which professionals are collaborating, the barrier of deciding who is best suited to perform specific components of patient care must be overcome.

\section{Solution}

Since the AT and PT have overlapping bodies of knowledge and skill sets, they would have frequent candid discussions focused on which professional was best suited to perform specific duties related to each patient. The responsibilities of each professional must be implemented in a manner that reflects the needs of the patient, the operational logistics of the clinic, the policies of the patient's insurance provider, and the licensing and practice acts of each professional. The PT and AT understood their own roles and the roles of the other, and used this knowledge appropriately to establish and meet patient goals. The PT and AT believed they had something to offer the patient, as well as offer the other profession [16]. It is important to believe that you have something to offer within your professional experience and expertise. When developing collaborative relationships, the PT and AT emphasized their strengths and how they could contribute to the patient.

\section{Barrier 5 \\ Discrepancy between professional reasoning}

There is often a discrepancy between the clinical reasoning of each professional attempting to collaborate [12]. When a team member offers insight and suggestions that goes against the logic or opinion of the other members, tension may develop which creates defensiveness. In hospital settings, health care professionals have identified that they were reluctant to express opinions during multidisciplinary team meetings for fear of being blamed for preventing a discharge from occurring [13]. Interprofessional differences in the management of the patient can cause discharge delays [13], such as when a PT and AT have differing opinions on the return-to-play status of a patient-athlete.

\section{Solution}

The PT and AT, at times, would have diverse opinions on how to achieve the goals of the patient. Because of the understanding that alternative schools of thought and clinical reasoning were a catalyst for 
professional development [6], the PT and AT were able to more easily see the gaps in their own clinical judgment and where their interventions were lacking when collaborating with other professionals. Discrepancies in professional logic can lead to improved patient safety and improved quality of care.

There is a relevance of the health history of the patients that are under the care of a PT or an AT. Every clinical intervention has to address the health condition, impairments, activity limitations and/or participation restrictions. However, virtually every rehabilitation intervention has risks, which may be magnified if the underlying health history, its severity and potential complications have not been properly evaluated. Examples include: manipulation of the spine in a patient with undiagnosed spinal malignancy or aplasia of dens axis; excessive movement of the hip joint after total hip replacement; soft tissue treatments under the condition of anticoagulants; attempted exercise in patients with inadequate cardiopulmonary reserve as a consequence of, say, ischemic heart disease; and, allowing an athlete to return to a sport prematurely with inadequate joint mobility and muscle strength. For this reason, a thorough examination and evaluation is essential prior to initiating a rehabilitation program, and a collaborative effort from an AT and a PT with different professional logic and opinions can make this examination and evaluation process more complete. Being open minded to alternative clinical decisionmaking models will not only improve a clinician's ability to make sound clinical judgments, but the quality of the patient's examination, evaluation, and treatment will be improved.

\section{Barrier 6}

\section{Territorial behavior}

Based on literature on organizations, leadership, and collaboration, territorial behavior among professional groups is a known barrier to interprofessional collaboration [7]. This is very common in many outpatient physical therapy practices, particularly among the owners and managers of these practices who regard their company as territories that they have to defend. With such a view, interprofessional collaboration may be perceived as a threat to their territorial control. Thus, instead of collaborating, outpatient physical therapy private practice owners and managers may use their energy and power to defend their territories [7]. In doing so, they may also defend their professional roles, competencies, and approaches against other professions and companies, which makes interprofessional collaboration very difficult [7]. Healthcare professionals should be motivated by concerns for their patients and not motivated by material rewards, privileges, or status $[7,17]$. If the boundaries or territories are unclear or disputed, this leads to struggles or conflicts between different professions and professionals. Many managers are expected to be a strong advocate for their company and to defend it like a territory. Professionalism by definition creates an independent framework focused on autonomy and control. Professionals with high levels of autonomy frequently work in a parallel fashion that is not conductive to team functioning [18].

\section{Solution}

Professionals engaged in collaboration may have less individual autonomy, however; the collaborative unit is more autonomous and has greater territorial control than professionals practicing independently [18]. The PT and AT, when they had a clear understanding of the goals of the collaboration and appropriately matched their individual roles and responsibilities to the collaborative goal, were able to manage any conflict that occurred with territorial behavior.

Practicing in a patient-centered fashion and focusing on the goals of the patient decreased the territorial behavior between the PT and AT. They frequently sought out, integrated, and valued the input of each professional when designing and implementing care plans with the primary goal of 
benefitting the patient. They did not automatically impose what they thought was helpful, but ensured open communication between the patient, the AT, and the PT. To collaborate across professional boundaries, especially between professionals with significant overlap, each professional must see beyond their own interests and be willing to sacrifice for the benefit of the patient [7]. Owners and managers of companies that employed the PT and AT played an important role in developing a culture of professional altruism within their company and within their brand.

\section{Barrier 7 \\ Ineffective communication}

Ineffective communication can contribute to or be a consequence of each of the previously discussed barriers. If each professional does not share critical information regarding the patient as well as issues related to decision-making, both the patient care and the collaborative relationship may suffer. The ability to present information in a manner that is relevant, concise, and timely is critical to the successful management of each patient and to the development of a collaborative relationship. Even timely, accurate information may not be heard or acted upon if the recipient does not respect the source [19]. During collaborative communication, if professionals do not feel free to voice their professional opinion and concerns, while not respectfully listening to the voices of other professionals, patient care will suffer $[20,21]$ and the collaborative relationship will not be able to be maintained.

\section{Solution}

Web-based technology assisted the PT and AT in sharing patient data and ideas. This occurred through email and text messaging, allowing them to share documents and information, as well as allowed for easy methods of communication. The interprofessional interactions between the PTs and AT were primarily business-like; however, they in- cluded social "small talk" during these interactions, especially involving humor. This provided a form of social cohesiveness and created an environment that encouraged free dialogue while reducing inherent threat of social contact [22].

The PT and AT ensured they had a means and a process for providing feedback to each other on their performance, which is necessary for professional development, quality of patient care, and sustainment of the collaborative relationship [22]. This occurred informally as a simple "thank you" or constructive comment after each professional updated the other on the progress of their patients. Informal communication included modes such as email, phone, and text messaging.

Formal communication occurred through weekly meetings they called "case conference" in which patient care of each patient was discussed. These meetings were open to all parties involved with the care of a patient. The "case conferences" were the main formal communication strategy used by this interprofessional team. These meetings afforded the professionals time to discuss patient management away from the frenetic environment of the outpatient orthopedic rehabilitation clinic. Workloads and patient schedules sometimes made these meetings difficult for both clinicians to attend; however, each meeting still took place even if the clinician leading the collaborative efforts for a specific patient was not present. This practice of meetings taking place without the "leader" helped to develop a culture that everyone's input was seen as crucial to decision-making. The PT or AT that was not able to attend the meeting was sent (via email) the meeting minutes as well as any patient notes required to get the absent party up to speed on the patient. If the PT or AT was not at a meeting in which urgent information had to be exchanged, then a phone call was made.

The Joint Commission on Accreditation of Healthcare Organizations (JCAHO) is a voluntary organization that monitors critical incidents and sentinel 
events in healthcare settings in the USA, which have been defined as "unexpected occurrences involving death or serious physical or psychological injury, or the risk thereof" [21]. In 2003, JCAHO reported that communication failures among team members are a contributory factor in $60 \%$ of sentinel events [21]. The factor most influential in reducing these events and their potentially negative effects on clinical outcomes is improvement of relationships among clinicians [21]. The "case conferences" between the AT and PT was a formal means to minimize communication failures and to develop and maintain a working relationship.

\section{Conclusion}

The complexity and quality of collaborative practice is beyond the control of any one professional, and the only efficient way to improve collaborative practice is to provide the other professionals with time, energy, knowledge, and encouragement to improve the collaborative relationship and benefit the patient. It is important to understand that building a successful collaborative relationship takes time and is a developmental process; thus, professionals cannot become interprofessional practitioners overnight. It appears that contextual factors, such as community in which the professionals practice, the healthcare setting, or the practice environment, have a far less important impact to successful collaboration than the professionals' attitudes and investment in the collaborative efforts [23].

Interprofessional practice is not a single, homogeneous treatment variable. Teams will vary in the quality of their functioning and collaboration is not a dichotomous variable, simply present or absent, but is present to varying degrees. The barriers described above are interconnected and the solutions cannot stand alone; therefore, successful interprofessional collaboration represents an integrated whole that relies on the interaction of each of the solutions mentioned to achieve improved patient care [4].
In the outpatient orthopedic setting, roles of a PT and an AT have an overlap of accountability. Adaptability and collaboration of clinicians, rather than adherence to strictly defined roles, has been found to capture errors and mitigate risk [6]. Collaboration between a PT and an AT can lead to power struggles and suboptimal patient care if these barriers are not overcome [6]; however, collaboration may be necessary to provide the highest quality of patient care.

A growing body of evidence supports the benefits of collaboration, including improved practitioner morale, greater job satisfaction, lower staff stress, improved patient satisfaction, and enhanced clinical effectiveness [6]. It is important to note that poor interprofessional collaboration can have a negative impact on the quality of patient care [20]. Thus, skills in working as an interprofessional team, gained through practice and experience, are important for high-quality care. It is challenging to link interprofessional collaboration with improved care because collaboration is a multi-dimensional construct. The interprofessional approach described in this report helps to demonstrate to importance of collaboration and may guide clinicians in their efforts to collaborate.

\section{Opportunities for Future Research}

While interprofessional collaboration research is still developing, it is encouraging that this research continues to grow [5]. Given the barriers to interprofessional collaboration, future research should focus on cost-benefit analysis, determining the optimal mix of collaborators, outline specific purposes of collaboration, and developing scales that measure degree of collaboration.

There are many challenges associated with this type of research, due to collaborative being a multidimensional construct. The few studies that were designed with a comparison or control group involved inconsistent outcomes across the studies, such as morbidity, mortality, functional outcomes, hospi- 
tal use, and costs [3]. Interprofessional practice was deemed 'more effective' in the majority of these studies; however, some studies showed that team care was more effective in some areas but not in others or that team care was judged to be 'as effective' for the outcomes examined [3]. It is noted that team care is often associated with increased use of some health services and that this increased use is associated with increased costs [3].

Three specific opportunities for research to expand the ideas presented in this paper include:

1) comparing patient outcomes and the costs required to achieve those outcomes in a collaborative practice and a non-collaborative practice

2) comparing the financial success of a collaborative practice with a non-collaborative practice

3) comparing patient outcomes in a collaborative practice with patient outcomes of this same practice after implementing the solutions discussed in this paper.

\section{Declaration of interest}

The authors report no conflicts of interest. The authors alone are responsible for the content and writing of the article.

\section{References}

1. Burwell. Setting Value-Based Payment Goals - HHS Efforts to Improve U.S. Health Care. N Engl J Med. 2015; 372(10):897-899. doi:10.1056/NEJMp1411429

2. Rice K, Zwarenstein M, Conn LG, Kenaszchuk C, Russell A, Reeves S. An intervention to improve interprofessional collaboration and communications: a comparative qualitative study. J Interprof Care. 2010; 24(4):350-361. doi:10.3109/13561820903550713

3. Schmitt $\mathrm{MH}$. Collaboration improves the quality of care: methodological challenges and evidence from US health care research. Journal of Interprofessional Care. 2009; 15(1):47-66. doi:10.1080/13561820020022873

4. Bainbridge LN, Orchard C, Wood V. Competencies for Interprofessional Collaboration. Journal of Physical Therapy Education. 2010; 24(1):6-11
5. ReevesS, Pelone F, Harrison R, Goldman J, Zwarenstein M. Interprofessional collaboration to improve professional practice and healthcare outcomes. Cochrane Database Syst Rev. 2017; 6:CD000072. doi:10.1002/14651858.CD000072.pub3

6. Yeager S. Interdisciplinary collaboration: the heart and soul of health care. Crit Care Nurs Clin North Am. 2005; 17(2):143-148. doi:10.1016/j.ccell.2005.01.003

7. Axelsson SB, Axelsson R. From territoriality to altruism in interprofessional collaboration and leadership. J Interprof Care. 2009; 23(4):320-330. doi:10.1080/13561820902921811

8. Gilbert JH, Yan J, Hoffman SJ. A WHO report: framework for action on interprofessional education and collaborative practice. J Allied Health. 2010. 39 Suppl 1:196-197.

9. Curley C, McEachern JE, Speroff T. A firm trial of interdisciplinary rounds on the inpatient medical wards: an intervention designed using continuous quality improvement. Med Care. 1998; 36(8 Suppl):AS4-12.

10. Arredondo P, Shealy C, Neale M, Winfrey LL. Consultation and interprofessional collaboration: modeling for the future. J Clin Psychol. 204; 60(7):787-800. doi:10.1002/jclp.20015

11. Riendeau C, Parent-Houle V, Lebel-Gabriel ME, Gauvin P, Liu le $Y$, Pearsonl, et al. An investigation of how university sports team athletic therapists and physical therapists experience ethical issues. J Orthop Sports Phys Ther. 2015; 45(3):198-206. doi:10.2519/jospt.2015.5390

12. Kvarnstrom S. Difficulties in collaboration: a critical incident study of interprofessional healthcare teamwork. J Interprof Care. 2008; 22(2):191-203. doi:10.1080/13561820701760600

13. Atwal A, Caldwell K. Do multidisciplinary integrated care pathways improve interprofessional collaboration? Scand J Caring Sci. 2002; 16(4):360-367.

14. Apker J, ProppKM, Zabava FordWS, Hofmeister N. Collaborati on,credibility,compassion, and coordination: professional nurse communication skill sets in health care team interactions. J Prof Nurs. 2006; 22(3):180-189.doi:10.1016/j.profnurs.2006.03.002

15. Way DJ, Busing N. Implementation Strategies: "Collaboration in Primary Care - Family Doctors \& Nurse Practitioners Delivering Shared Care". Ontario College of Family Physicians. 2000.

16. Fell DK. Developing International Collaborations in Physical Therapy Academia: A Case Example Emphasizing Education and Scholarship. Journal of Physical Therapy Education. 2012; 26(1):6-12.

17. Johnson P, Wistow G, Schulz R, Hardy B. Interagency and interprofessional collaboration in community care: the interdependence of structures and values. J Interprof Care. 2003; 17(1):69-83.

18. Rose L. Interprofessional collaboration in the ICU: how to define? Nurs Crit Care. 2011; 16(1):5-10. doi:10.1111/j.14785153.2010.00398.x 
19. Panel IECE. Core Competencies for Interprofessional Collaborative Practice: Report of an Expert Panel. 2011; Paper presented at the Interprofessional Education Collaborative, Washington, D.C.

20. Bridges DR, Davidson RA, Odegard PS, Maki IV, Tomkowiak J. Interprofessional collaboration: three best practice models of interprofessional education. Med Educ Online. 2011; 16. doi:10.3402/meo.v16i0.6035

21. Martin JS, Ummenhofer W, Manser T, Spirig R. Interprofessional collaboration among nurses and physicians: making a difference in patient outcome. Swiss Med Wkly. 2010; 140:w13062. doi:10.4414/smw.2010.13062

22. Reeves S, Lewin S. Interprofessional collaboration in the hospital: strategies and meanings. J Health Serv Res Policy. 2004; 9(4):218-225. doi:10.1258/1355819042250140

23. Sicotte C, D'Amour D, Moreault MP. Interdisciplinary collaboration within Quebec Community Health Care Centres. Soc Sci Med. 2002; 55(6):991-1003.

Publish in International Archives of Medicine

International Archives of Medicine is an open access journal publishing articles encompassing all aspects of medical science and clinical practice. IAM is considered a megajournal with independent sections on all areas of medicine. IAM is a really international journal with authors and board members from all around the world. The journal is widely indexed and classified Q2 in category Medicine. 